\title{
Anxiety and pre-symptomatic testing for neurodegenerative disorders
}

\author{
Lêdo Susana ${ }^{1,2,3^{*}}$, Leite Ângela ${ }^{1,4,5}$, Jorge Sequeiros ${ }^{1,2}$ \\ ${ }^{1}$ Centre for Predictive and Preventive Genetics (CGPP), IBMC - Institute for Molecular and Cell Biology, Porto, Portugal \\ ${ }^{2}$ ICBAS, Universidade do Porto, Porto, Portugal \\ ${ }^{3}$ ISCS-Norte (CESPU), Porto, Portugal \\ ${ }^{4}$ ISCET - Instituto Superior de Ciências Empresariais e do Turismo, Porto, Portugal \\ ${ }^{5}$ ULP-Universidade Lusófona do Porto, Porto, Portugal \\ Email: ${ }^{\text {susanaledo@gmail.com }}$
}

Received 17 May 2013; revised 2 June 2013; accepted 10 July 2013

Copyright (C) 2013 Lêdo Susana et al. This is an open access article distributed under the Creative Commons Attribution License, which permits unrestricted use, distribution, and reproduction in any medium, provided the original work is properly cited.

\begin{abstract}
In this retrospective study we have investigated the anxiety as an impact of pre-symptomatic testing (PST) for 3 autosomal dominant late-onset diseases: Huntington disease (HD), Machado-Joseph disease (MJD) and familial amyloidotic polyneuropathy (FAP) V30M TTR. The study included 686 subjects: 586 (85.4\%) were the offspring at risk for FAP, $92(13.4 \%)$ for HD and $8(1.2 \%)$ to MJD. Of these, 352 received the carrier result and 305 the non-carrier result. As indicator of anxiety distress was taken the Self-Rating Anxiety Scale of Zung (SAS), applied in the pre-test and the three post-test moments: three weeks, 6 months and one year after notification of test results. Values decreased significantly along the four evaluation moments, regardless the studied disease or test result. For female population, SAS means cores revealed results of clinical anxiety at pre-test, only decreasing to non clinical scores a year after PST disclosure.
\end{abstract}

Keywords: Anxiety; Subscales; SAS; Psychological Impact; FAP; HD; MJD

\section{INTRODUCTION}

There are numerous diagnostic or pre-symptomatic tests (PST) for hereditary diseases [1-3] Machado-Joseph disease (MJD) or amyloidotic polyneuropathy (FAP) TTR V30M, all late onset autosomal dominant diseases, the PST can predict if, in future more or less distant, the person will develop the symptoms of the disease $[2,4]$.

It's in this field of monogenic autosomal dominant late onset diseases, that the Center for Predictive and Preven-

*Corresponding author. tive Genetics (CGPP) at Institute for Molecular and Cell Biology (IBMC), Oporto University, provides a multidisciplinary approach for HD, PAF and MJD PST.

A Predictive protocol for neurodegenerative diseases implemented in CGPP is a national reference model for genetic counseling and psychosocial support for people at risk of suffering such progressive and debilitating diseases without effective treatment and cure until the present days [2].

\section{The Studied Diseases}

HD, MJD and FAP are three examples of monogenic autosomal dominant of late onset, clinically considered as neurodegenerative diseases, incurable and highly debilitating and that may take a broad spectrum of symptomatic manifestations.

Huntington's disease $[5,6]$ is the most studied, largely due to the discovery by Guselli and colleagues, of its genetic marker since 1983 [7]. Thus, the predictive test for Huntington's disease began to be held in Canada in 1986 and US $[1,8]$, with over the 90's progress and the new laboratory techniques for mutation detection $[5,6]$.

MJD and FAP are very specific Portuguese diseases, that also have a severe neurodegenerative pathway, and for which there is still no effective treatment or cure. In 1993, the MJD gene was finally located on chromosome 14 by a group of researchers led by Shoji Tsuji, later confirmed in the Portuguese households by Sequeiros and colleagues [9]. The genetic mutation present in FAP leads to the production of an amyloid protein, immunologically related to transthyretin (TTR) that is abnormally degraded, precipitated and stored in tissues as amyloid substance [10], deposited in the tissues of various organs leading these patients for a progressive limitation $[11,12]$. 
Pre-symptomatic diagnosis is available since 1984 [11]. Several psychosocial studies have been done with families and their descendants at risk for neurodegenerative diseases diagnosed in CGPP [13-15].

Lêdo [13] studied FAP carriers after a year of knowledge of their genetic status, and concluded there had been no presence of emotional distress and feelings of hopelessness.

Other studies with subjects at risk for FAP, HD and MJD pointed to the existence of psychological well-being and better health perception than the control subjects [14]. Also in this field, there have been published psychosocial genetic studies related to the experience of more than 10 years in the counseling of individuals at risk [16], as well as studies about the importance of contact time with the disease or affected father figure in the psychological impact of PST [15].

Despite the different approaches that have been made, there are still issues to be elucidated regarding the impact of the application of PST to diseases with common starting symptoms at the early adulthood and a degenerative path, but with different treatment options and clinical outcomes (e.g. the psychiatric disorders, unique to Huntington's disease, and for MJD frequent signs of cerebellar ataxia, progressive external ophthalmoplegia and pyramidal signs $[9,17,18]$.

On the other hand, it continues to be relevant studies related to psychological impact of test results, mainly the anxiety indexes because this is one of the most expressed feelings at the first evaluation. In this sense, we established as objectives of this research: 1) the anxiety indexes observed before and after completion of the PST, and 2) the differences on anxiety indexes related to types of disease, carrier or non carrier status, and some demographic variables.

\section{MATERIAL AND METHODS}

It was designed a retrospective study of clinical files of subjects who underwent pre-symptomatic testing for genetic autosomal dominant diseases with late onset (MJD, HD and FAP), in CGPP, between 2000 and 2010. These files contained psychological evaluations data conducted along the four moments of the general psychological evaluation protocol: 1) $1^{\text {st }}$ moment, pre-test, prior to the genetic test; 2) $2^{\text {nd }}$ moment of evaluation, three weeks after receiving the test result and knowing genetic status; 3 ) $3^{\text {rd }}$ moment, six months after disclosure; 4) $4^{\text {th }}$ moment, one year after disclosure.

\subsection{Subjects}

The initial sample (Table 1) is constituted by 686 subjects at base line: $586(85.4 \%)$ attended the service to accomplish the pre-symptomatic test for FAP, 92 (13.4\%) for HD and $8(1.2 \%)$ for MJD. Subjects underwent the evaluation protocol voluntarily when they were informed they were $50 \%$ at-risk for these diseases and were informed about the purpose of the research, simultaneously with PST protocol procedure and signed a written consent for the use of their data with the finality of scientific research. $58.6 \%$ of the complete sample were women. It was found that $51.3 \%$ of the subjects were single, $44.7 \%$ were married. Of the total initial subjects, 29 did not appear to know their test result, $352(51.6 \%)$ received the result of carriers and $305(44.7 \%)$ received the result of non-carriers. Along the four moment of the general protocol, subjects were given up. This is why we witnessed a sharp decline in the subject number at the post-test one year later.

Men and women have proven to be equivalent in their distribution regarding the age $\left(\mathrm{X}^{2}=636.939 ; \mathrm{df}=625 ; \mathrm{p}\right.$

Table 1. Sample characteristics along the four moments of the general psychological evaluation protocol.

\begin{tabular}{|c|c|c|c|c|c|c|c|c|c|c|c|c|}
\hline & \multicolumn{3}{|c|}{ Pre-test a $(\mathrm{N}=686)$} & \multicolumn{3}{|c|}{ Post-test b $(\mathrm{N}=290)$} & \multicolumn{3}{|c|}{ Post-test c $(\mathrm{N}=143)$} & \multicolumn{3}{|c|}{ Post-test d $(\mathrm{N}=54)$} \\
\hline & FAP & HD & MJD & FAP & HD & MJD & FAP & HD & MJD & FAP & HD & MJD \\
\hline $\mathbf{N}$ & 586 & 92 & 8 & 248 & 38 & 4 & 114 & 25 & 4 & 40 & 13 & 1 \\
\hline Gender & $\begin{array}{c}\text { F } 340 \\
\text { M } 246\end{array}$ & $\begin{array}{c}\text { F } 54 \\
\text { M } 38\end{array}$ & $\begin{array}{l}\text { F } 8 \\
\text { M } 0\end{array}$ & $\begin{array}{l}\text { F } 146 \\
\text { M } 102\end{array}$ & $\begin{array}{l}\text { F } 20 \\
\text { M } 18\end{array}$ & $\begin{array}{l}\text { F } 4 \\
\text { M } 0\end{array}$ & $\begin{array}{l}\text { F } 64 \\
\text { M } 50\end{array}$ & $\begin{array}{l}\text { F } 14 \\
\text { M } 11\end{array}$ & $\begin{array}{l}\text { F } 4 \\
\text { M } 0\end{array}$ & $\begin{array}{l}\text { F } 25 \\
\text { M } 15\end{array}$ & $\begin{array}{l}\text { F } 7 \\
\text { M } 6\end{array}$ & $\begin{array}{l}\text { F } 1 \\
\text { M } 0\end{array}$ \\
\hline Mean Age & 35.09 & 43.69 & 38.75 & 34.83 & 46.45 & 48.00 & 34.68 & 45.24 & 48.00 & 31.85 & 45.46 & 37.00 \\
\hline $\begin{array}{c}\text { Marital } \\
\text { Status }\end{array}$ & $\begin{array}{c}\text { S } 320 \\
\text { M } 239 \\
\text { D } 10 \\
\text { W } 8\end{array}$ & $\begin{array}{c}\text { S } 27 \\
\text { M } 59 \\
\text { D } 0 \\
\text { W } 0\end{array}$ & $\begin{array}{l}\text { S } 2 \\
\text { M } 6 \\
\text { D } 0 \\
\text { W } 0\end{array}$ & $\begin{array}{c}\text { S } 134 \\
\text { M } 104 \\
\text { D } 3 \\
\text { W } 3\end{array}$ & $\begin{array}{c}\text { S } 13 \\
\text { M } 23 \\
\text { D } 1 \\
\text { W } 1\end{array}$ & $\begin{array}{c}\text { S } 1 \\
\text { M } 3 \\
\text { D } 0 \\
\text { W } 0\end{array}$ & $\begin{array}{c}\text { S } 59 \\
\text { M } 52 \\
\text { D } 0 \\
\text { W } 1\end{array}$ & $\begin{array}{c}\text { S } 9 \\
\text { M } 14 \\
\text { D } 1 \\
\text { W } 1\end{array}$ & $\begin{array}{l}\text { S } 1 \\
\text { M } 3 \\
\text { D } 0 \\
\text { W } 0\end{array}$ & $\begin{array}{l}\text { S } 22 \\
\text { M } 17 \\
\text { D } 0 \\
\text { W } 0\end{array}$ & $\begin{array}{c}\text { S } 2 \\
\text { M } 11 \\
\text { D } 0 \\
\text { W } 0\end{array}$ & $\begin{array}{c}\text { S } 0 \\
\text { M } 1 \\
\text { D } 0 \\
\text { W } 0\end{array}$ \\
\hline Test Result & $\begin{array}{c}\text { NC } 311 \\
\text { C } 254 \\
\text { DK } 17\end{array}$ & $\begin{array}{c}\text { NC } 39 \\
\text { C } 45 \\
\text { DK } 8\end{array}$ & $\begin{array}{c}\mathrm{NC} 2 \\
\mathrm{C} 6 \\
\mathrm{DK} 0\end{array}$ & $\begin{array}{c}\text { NC } 124 \\
\text { C } 117 \\
\text { DK } 5\end{array}$ & $\begin{array}{c}\text { NC } 16 \\
\text { C } 21 \\
\text { DK } 1\end{array}$ & $\begin{array}{c}\mathrm{NC} 0 \\
\mathrm{C} 4 \\
\mathrm{DK} 0\end{array}$ & $\begin{array}{c}\text { NC } 47 \\
\text { C } 62 \\
\text { DK } 3\end{array}$ & $\begin{array}{l}\text { NC } 5 \\
\text { C } 19 \\
\text { DK } 1\end{array}$ & $\begin{array}{c}\mathrm{NC} 0 \\
\mathrm{C} 4 \\
\mathrm{DK} 0\end{array}$ & $\begin{array}{c}\text { NC } 10 \\
\text { C } 29 \\
\text { DK } 0\end{array}$ & $\begin{array}{c}\mathrm{NC} 2 \\
\mathrm{C} 9 \\
\mathrm{DK} 2\end{array}$ & $\begin{array}{c}\mathrm{NC} 0 \\
\mathrm{C} 1 \\
\mathrm{DK} 0\end{array}$ \\
\hline
\end{tabular}

Gender (Female; Male); Marital Status (Single; Married; Divorced; Widow); Test Result (Non Carrier; Carrier; Don't know). 
$=0.362)$, marital status $\left(X^{2}=5.733 ; \mathrm{df}=2 ; \mathrm{p}=0.057\right)$, and test result $\left(X^{2}=2.446 ; d f=2 ; p=0.294\right)$.

\subsection{Procedure}

The PST protocol queries for neurodegenerative diseases in CGPP have been published elsewhere [2].

In the context of the protocol, each subject answered the anxiety scale evaluation along four stages: 1 ) pre-test: the first psychological evaluation, it was done the survey and evaluation of the motivations that led the person to pre-symptomatic test, exploring his/her own decision making processes and detection of emotional states that might jeopardize a good adjustment to the predictive test result (hereafter designated $1^{\text {st }}$ moment); 2) post-test: three weeks after receiving the test result post-test (hereafter designated $2^{\text {nd }}$ moment); 3 ) six months after disclosure (hereafter designated $3^{\text {rd }}$ moment); 4) one year after reporting the genetic test result (hereafter designated $4^{\text {th }}$ moment).

The socio-demographic variables (gender, age and marital status) and medical history were collected at the first psychological evaluation.

The anxiety variable was collected by the application of the Portuguese version [19] of the Self-Rating Anxiety Scale of Zung (SAS) [20]. This scale is composed of 20 items rated on a Likert scale of four grades (1 "rarely or never" to 4 "most or all of the time") and measure the anxiety clinical symptoms.

Anxiety is evaluated from the description of its most common symptoms and signals through four anxiety components (subscales): cognitive (items $1-3,4$ and 5) which can reach a maximum of 20 points, motor (items 6 , 7,8 and 9) which can reach a maximum of 16 , vegetative (items $10-16,17$ and 18) that can reach a maximum of 36 and central nervous system-CNS - (items 19 and 20) with a maximum value of 8 points. The score ranges between 20 and 80 and the cut point is 40 [19].

\subsection{Data Analysis}

The statistical analysis was performed with the software PASW Statistics 19.0 [21]. We carry out procedures related to descriptive statistics (frequencies, mean, standard deviation, minimum, maximum), bi-variate statistics (mean, ANOVA, correlation bi-varied); prediction of numerical results (multiple linear regression, stepwise) predicting for the identification of groups (factor analysis and discriminant analysis).

\section{RESULTS}

\subsection{Descriptive Analysis for the Four Evaluation Moments}

We analyzed the mean and standard deviation of the results obtained from the application of SAS in the four moments considered, for the total sample and for female and male subsamples.

Reading Table 2, it can be seen that, along the four moments, women had always higher averages than men. For both genders, $1^{\text {st }}$ moment revealed higher mean values (male: $\mathrm{M}=39.6, \mathrm{SD}=8.07$; female: $\mathrm{M}=43.7, \mathrm{SP}=$ 8.87), however, in women, there is a slight increase in average from the second to the third moment of data collection.

\subsection{Descriptive Statistics of the 20 SAS Items for the Four Stages of Evaluation}

We proceeded to the descriptive analysis for the 20 items of the scale (mean, standard deviation and percentage of symptomatic responses, i.e., those scored with 2,3 or 4 points) for the total sample and male and female subsamples, for four moment's evaluation. We can see that, at first assessment (Table 3), i.e., before genetic testing $\left(1^{\text {st }}\right.$ moment), women, in general, have significantly higher averages in some of the items that described anxiety symptoms, for example, restlessness and fear, headaches, neck and back pain and stomachache, or more nightmares.

At post-test, three weeks after the communication of the PST result $\left(2^{\text {nd }}\right.$ moment), it was found, with statistically significant results, that women continued to show a greater tendency to feel more nervous and anxious than men and have higher sleep disturbance (Table 4).

Regarding Table 5, six months after knowing test result $\left(3^{\text {rd }}\right.$ moment), women fell more scared for no reason and have hands dry and warm more often than men; furthermore, men fell that things will be all right more than women.

Reading Table 6, at $4^{\text {th }}$ moment, there are no statistically significant differences between female and male subsamples.

\subsection{Comparison of the Total Means along the Four Evaluation Moments}

We found that the for the total anxiety average decreased over the four moments. In almost all moments compared, except between the $1^{\text {st }}$ and the $3^{\text {rd }}$ moments, and the $2^{\text {nd }}$ and $3^{\text {rd }}$ moments, the mean values obtained from the application of SAS decreased significantly with a $\mathrm{p}$ value less than 0.05 (see Table 7).

\subsection{Comparison of the SAS Subscales Means along the Four Evaluation Moments}

We observed a decrease in all SAS subscales over the four moments of our evaluation as we can see from the reading Tables 8-11.

In Table 8, we found statistically significant differences between $1^{\text {st }}$ and $2^{\text {nd }}$ moments, $1^{\text {st }}$ and $3^{\text {rd }}$ moments, 
and $2^{\text {nd }}$ and $3^{\text {rd }}$ moments regarding motor anxiety subscale.

In Table 9, we found statistically significant differences between $1^{\text {st }}$ and $2^{\text {nd }}$ moments, and $1^{\text {st }}$ and $4^{\text {th }}$ moments regarding cognitive anxiety subscale.

In Table 10, we found statistically significant differ- ences between $1^{\text {st }}$ and $2^{\text {nd }}$ moments, $1^{\text {st }}$ and $4^{\text {th }}$ moments, and $3^{\text {rd }}$ and $4^{\text {th }}$ moments regarding vegetative anxiety subscale.

In Table 11, we found statistically significant differences between $1^{\text {st }}$ and $3^{\text {rd }}$ moments, and $1^{\text {st }}$ and $4^{\text {th }}$ moments regarding CNS anxiety subscale.

Table 2. Total SAS results (mean, standard deviation) for the four moments evaluated $\left(1^{\text {st }}, 2^{\text {nd }}, 3^{\text {rd }}, 4^{\text {th }}\right)$, for the total sample and female and male subsamples.

\begin{tabular}{|c|c|c|c|c|c|c|}
\hline & \multicolumn{2}{|c|}{ Total Sample } & \multicolumn{2}{|c|}{ Female } & \multicolumn{2}{|c|}{ Male } \\
\hline & $M$ & $S D$ & $M$ & $S D$ & $M$ & $S D$ \\
\hline SAS & 41.9 & 8.75 & 43.7 & 8.87 & 39.6 & 8.07 \\
\hline $1^{\text {st }}$ moment & \multicolumn{2}{|c|}{$(n=653 ; \alpha=0.80)$} & \multicolumn{2}{|c|}{$(n=378 ; \alpha=0.79)$} & \multicolumn{2}{|c|}{$(n=275 ; \alpha=0.78)$} \\
\hline SAS & 40.2 & 8.21 & 41.4 & 8.45 & 38.6 & 7.61 \\
\hline $2^{\text {nd }}$ moment & \multicolumn{2}{|c|}{$(n=232 ; \alpha=0.82)$} & \multicolumn{2}{|c|}{$(n=135 ; \alpha=0.82)$} & \multicolumn{2}{|c|}{$(n=97 ; \alpha=0.79)$} \\
\hline SAS & 41.0 & 9.87 & 43.0 & 10.10 & 38.4 & 9.05 \\
\hline $3^{\text {rd }}$ moment & \multicolumn{2}{|c|}{$(n=85 ; \alpha=0.83)$} & \multicolumn{2}{|c|}{$(n=48 ; \alpha=0.82)$} & \multicolumn{2}{|c|}{$(n=37 ; \alpha=0.85)$} \\
\hline SAS & 36.7 & 8.10 & 37.2 & 7.40 & 35.8 & 9.02 \\
\hline $4^{\text {th }}$ moment & \multicolumn{2}{|c|}{$(n=62 ; \alpha=0.83)$} & \multicolumn{2}{|c|}{$(n=35 ; \alpha=0.79)$} & \multicolumn{2}{|c|}{$(n=27 ; \alpha=0.87)$} \\
\hline
\end{tabular}

Table 3. Results of the items of the SAS $1^{\text {st }}$ moment (mean, standard deviation, and percentage of symptomatic responses) for the total sample and female and male subsamples.

\begin{tabular}{|c|c|c|c|c|c|c|c|c|c|}
\hline \multirow{2}{*}{ Item } & \multicolumn{3}{|c|}{ Total Sample } & \multicolumn{3}{|c|}{ Female } & \multicolumn{3}{|c|}{ Male } \\
\hline & $M$ & $S D$ & $\%$ & $M$ & $S D$ & $\%$ & $M$ & $S D$ & $\%$ \\
\hline 1-I feel more nervous and anxious than usual. ${ }^{* *}$ & 1.86 & 0.82 & 62.6 & 1.96 & 0.80 & 70.4 & 1.72 & 0.83 & 51.4 \\
\hline 2-I feel afraid for no reason. ${ }^{*}$ & 1.39 & 0.65 & 31.1 & 1.44 & 0.66 & 35.9 & 1.32 & 0.63 & 24.4 \\
\hline 3-I get upset easily or feel panicky. & 1.22 & 0.53 & 17.7 & 1.24 & 0.55 & 19.0 & 1.20 & 0.50 & 15.2 \\
\hline 4-I feel like I'm falling apart and going to pieces. & 1.40 & 0.66 & 31.3 & 1.43 & 0.66 & 34.8 & 1.35 & 0.65 & 26.4 \\
\hline 5-I feel that everything is all right and nothing bad will happen. ${ }^{* *}$ & 2.58 & 0.95 & 85.6 & 2.67 & 0.93 & 88.0 & 2.46 & 0.97 & 82.0 \\
\hline 6-My arms and legs shake and tremble." & 1.42 & 0.62 & 35.9 & 1.47 & 0.62 & 40.4 & 1.35 & 0.61 & 29.7 \\
\hline 7—I am bothered by headaches neck and back pain.* & 1.70 & 0.77 & 54.1 & 1.80 & 0.79 & 60.6 & 1.56 & 0.71 & 45.2 \\
\hline 8-I feel weak and get tired easily. ${ }^{*}$ & 1.44 & 0.67 & 34.6 & 1.49 & 0.72 & 37.1 & 1.36 & 0.59 & 31.1 \\
\hline 9-I feel calm and can sit still easily. ${ }^{* *}$ & 2.41 & 1.04 & 76.7 & 2.51 & 1.02 & 81.2 & 2.26 & 1.04 & 70.6 \\
\hline 10-I can feel my heart beating fast. & 1.42 & 0.62 & 357 & 1.45 & 0.63 & 38.2 & 1.37 & 0.60 & 32.2 \\
\hline $11-$ I am bothered by dizzy spells. & 1.25 & 0.52 & 21.1 & 1.27 & 0.53 & 23.9 & 1.21 & 0.52 & 17.2 \\
\hline 12 - I have fainting spells or feel like it. & 1.08 & 0.32 & 7.2 & 1.10 & 0.36 & 8.9 & 1.06 & 0.26 & 5.0 \\
\hline 13 - I can breathe in and out easily. ${ }^{* *}$ & 1.70 & 1.02 & 38.5 & 1.79 & 1.05 & 42.7 & 1.57 & 0.95 & 31.8 \\
\hline 14-I get feelings of numbness and tingling in my fingers \& toes. & 1.36 & 0.64 & 29.3 & 1.39 & 0.67 & 30.6 & 1.33 & 0.58 & 27.5 \\
\hline 15-I am bothered by stomach aches or indigestion.* & 1.43 & 0.70 & 33.6 & 1.50 & 0.75 & 38.7 & 1.33 & 0.61 & 26.8 \\
\hline 16-I have to empty my bladder often. & 1.75 & 0.78 & 56.6 & 1.78 & 0.81 & 57.9 & 1.70 & 0.75 & 54.6 \\
\hline 17-My hands are usually dry and warm. & 2.81 & 1.09 & 83.4 & 2.87 & 1.08 & 85.1 & 2.74 & 1.10 & 81.1 \\
\hline 18-My face gets hot and blushes. ${ }^{* *}$ & 1.95 & 0.86 & 66.8 & 2.08 & 0.91 & 71.5 & 1.77 & 0.76 & 60.2 \\
\hline 19-I fall asleep easily and get a good night's rest. ${ }^{* *}$ & 1.98 & 1.05 & 55.5 & 2.07 & 1.07 & 58.9 & 1.85 & 1.00 & 50.7 \\
\hline 20-I have nightmares. ${ }^{* *}$ & 1.39 & 0.66 & 31.6 & 1.46 & 0.70 & 36.4 & 1.30 & 0.58 & 24.9 \\
\hline
\end{tabular}

Note: ${ }^{*}$ Differences between men and women to $\mathrm{p}<0.05 ;{ }^{* *}$ Differences between men and women to $\mathrm{p}<0.01$. 
Table 4. Results of the items of the SAS $2^{\text {nd }}$ moment (mean, standard deviation, and percentage of symptomatic responses) for the total sample and female and male subsamples.

\begin{tabular}{|c|c|c|c|c|c|c|c|c|c|}
\hline \multirow{2}{*}{ Item } & \multicolumn{3}{|c|}{ Total Sample } & \multicolumn{3}{|c|}{ Female } & \multicolumn{3}{|c|}{ Male } \\
\hline & $M$ & $S D$ & $\%$ & $M$ & $S D$ & $\%$ & $M$ & $S D$ & $\%$ \\
\hline 1-I feel more nervous and anxious than usual. ${ }^{*}$ & 1.58 & 0.73 & 45.4 & 1.66 & 0.78 & 50.5 & 1.46 & 0.65 & 38.1 \\
\hline 2-I feel afraid for no reason. & 1.32 & 0.58 & 26.1 & 1.36 & 0.62 & 29.4 & 1.25 & 0.51 & 21.5 \\
\hline 3-I get upset easily or feel panicky. & 1.20 & 0.45 & 17.9 & 1.21 & 0.46 & 18.9 & 1.18 & 0.43 & 16.6 \\
\hline 4-I feel like I'm falling apart and going to pieces. & 1.32 & 0.59 & 26.2 & 1.34 & 0.63 & 27.2 & 1.29 & 0.54 & 24.8 \\
\hline 5-I feel that everything is all right and nothing bad will happen. ${ }^{*}$ & 2.46 & 0.93 & 83.1 & 2.56 & 0.90 & 86.5 & 2.31 & 0.95 & 78.5 \\
\hline 6-My arms and legs shake and tremble. & 1.33 & 0.54 & 30.0 & 1.36 & 0.55 & 32.6 & 1.30 & 0.53 & 26.4 \\
\hline 7- I am bothered by headaches neck and back pain. & 1.64 & 0.73 & 50.6 & 1.66 & 0.74 & 51.8 & 1.60 & 0.71 & 48.8 \\
\hline 8 - I feel weak and get tired easily. & 1.43 & 0.70 & 33.0 & 1.46 & 0.76 & 33.0 & 1.39 & 0.61 & 33.0 \\
\hline 9-I feel calm and can sit still easily. & 2.39 & 1.00 & 75.9 & 2.48 & 0.96 & 80.0 & 2.26 & 1.04 & 69.3 \\
\hline $10-$ I can feel my heart beating fast. & 1.37 & 0.57 & 32.3 & 1.40 & 0.58 & 35.3 & 1.33 & 0.57 & 28.1 \\
\hline $11-\mathrm{I}$ am bothered by dizzy spells. & 1.27 & 0.55 & 22.1 & 1.29 & 0.62 & 21.8 & 1.23 & 0.42 & 22.5 \\
\hline 12-I have fainting spells or feel like it. & 1.09 & 0.32 & 7.9 & 1.11 & 0.37 & 9.4 & 1.06 & 2.23 & 5.8 \\
\hline 13-I can breathe in and out easily. & 1.59 & 0.96 & 33.6 & 1.58 & 0.97 & 31.7 & 1.60 & 0.94 & 36.4 \\
\hline $14-$ I get feelings of numbness and tingling in my fingers \& toes. & 1.28 & 0.53 & 24.1 & 1.25 & 0.52 & 21.2 & 1.32 & 0.53 & 28.3 \\
\hline 15-I am bothered by stomach aches or indigestion. & 1.43 & 0.65 & 35.4 & 1.44 & 0.64 & 35.9 & 1.43 & 0.66 & 34.7 \\
\hline 16-I have to empty my bladder often. & 1.71 & 0.75 & 55.6 & 1.72 & 0.77 & 55.3 & 1.70 & 0.72 & 55.8 \\
\hline 17-My hands are usually dry and warm. & 2.82 & 1.09 & 83.6 & 2.84 & 1.05 & 85.2 & 2.80 & 1.15 & 80.1 \\
\hline 18-My face gets hot and blushes. & 1.84 & 0.79 & 64.0 & 1.89 & 0.87 & 63.3 & 1.77 & 0.67 & 65.0 \\
\hline 19-I fall asleep easily and get a good night's rest." & 1.97 & 1.01 & 57.0 & 2.08 & 1.02 & 62.3 & 1.82 & 0.98 & 49.6 \\
\hline 20-I have nightmares. ${ }^{*}$ & 1.34 & 0.60 & 28.2 & 1.40 & 0.67 & 31.2 & 1.26 & 0.47 & 24.0 \\
\hline
\end{tabular}

Note: ${ }^{*}$ Differences between men and women to $\mathrm{p}<0.05$.

Table 5. Results of the items of the SAS $3^{\text {rd }}$ moment (mean, standard deviation, and percentage of symptomatic responses) for the total sample and female and male subsamples.

\begin{tabular}{|c|c|c|c|c|c|c|c|c|c|}
\hline \multirow{2}{*}{ Item } & \multicolumn{3}{|c|}{ Total Sample } & \multicolumn{3}{|c|}{ Female } & \multicolumn{3}{|c|}{ Male } \\
\hline & $M$ & $S D$ & $\%$ & $M$ & $S D$ & $\%$ & $M$ & $S D$ & $\%$ \\
\hline 1-I feel more nervous and anxious than usual. & 1.77 & 0.76 & 57.7 & 1.81 & 0.75 & 61.3 & 1.71 & 0.78 & 53.2 \\
\hline 2-I feel afraid for no reason. ${ }^{*}$ & 1.29 & 0.59 & 21.7 & 1.38 & 0.66 & 27.5 & 1.17 & 0.46 & 14.3 \\
\hline 3-I get upset easily or feel panicky. & 1.25 & 0.54 & 19.4 & 1.26 & 0.53 & 22.3 & 1.22 & 0.56 & 15.5 \\
\hline 4-I feel like I'm falling apart and going to pieces. & 1.43 & 0.63 & 35.1 & 1.49 & 0.64 & 40.8 & 1.34 & 0.61 & 27.6 \\
\hline 5-I feel that everything is all right and nothing bad will happen.* & 2.37 & 0.92 & 82.1 & 2.54 & 0.92 & 88.2 & 2.14 & 0.89 & 74.2 \\
\hline 6-My arms and legs shake and tremble. & 1.36 & 0.57 & 31.4 & 1.41 & 0.61 & 34.2 & 1.29 & 0.50 & 27.6 \\
\hline 7- I am bothered by headaches neck and back pain. & 1.69 & 0.78 & 52.6 & 1.69 & 0.79 & 52.0 & 1.69 & 0.75 & 53.5 \\
\hline 8 - I feel weak and get tired easily. & 1.40 & 0.63 & 32.1 & 1.43 & 0.62 & 36.9 & 1.34 & 0.64 & 25.8 \\
\hline 9-I feel calm and can sit still easily. & 2.11 & 0.95 & 69.4 & 2.09 & 0.91 & 71.1 & 2.14 & 1.02 & 67.3 \\
\hline $10-$ I can feel my heart beating fast. & 1.37 & 0.51 & 35.1 & 1.43 & 0.55 & 40.8 & 1.28 & 0.45 & 27.6 \\
\hline 11 - I am bothered by dizzy spells. & 1.36 & 0.64 & 27.5 & 1.36 & 0.67 & 26.3 & 1.36 & 0.61 & 29.3 \\
\hline 12-I have fainting spells or feel like it. & 1.13 & 0.47 & 9.7 & 1.20 & 0.59 & 13.1 & 1.05 & 0.22 & 5.2 \\
\hline 13 - I can breathe in and out easily. & 1.66 & 0.95 & 39.6 & 1.71 & 0.99 & 42.1 & 1.59 & 0.90 & 36.3 \\
\hline 14-I get feelings of numbness and tingling in my fingers \& toes. & 1.37 & 0.66 & 29.1 & 1.29 & 0.63 & 21.1 & 1.48 & 0.68 & 39.7 \\
\hline 15-I am bothered by stomach aches or indigestion. & 1.42 & 0.64 & 35.1 & 1.46 & 0.66 & 38.2 & 1.36 & 0.61 & 31.0 \\
\hline 16-I have to empty my bladder often. & 1.81 & 0.82 & 58.9 & 1.87 & 0.81 & 63.1 & 1.74 & 0.85 & 53.4 \\
\hline 17-My hands are usually dry and warm.* & 2.81 & 1.12 & 81.9 & 3.05 & 1.06 & 88.2 & 2.49 & 1.14 & 73.7 \\
\hline 18-My face gets hot and blushes. & 1.75 & 0.80 & 56.7 & 1.79 & 0.85 & 57.9 & 1.71 & 0.73 & 55.2 \\
\hline 19-I fall asleep easily and get a good night's rest. & 1.84 & 0.99 & 50.0 & 1.91 & 0.97 & 55.3 & 1.76 & 1.01 & 43.1 \\
\hline 20-I have nightmares. & 1.37 & 0.66 & 29.8 & 1.45 & 0.70 & 35.4 & 1.28 & 0.56 & 22.4 \\
\hline
\end{tabular}

Note: ${ }^{*}$ Differences between men and women to $\mathrm{p}<0.05 ;{ }^{* *}$ Differences between men and women to $\mathrm{p}<0.01$. 
Table 6. Results of the items of the SAS d (mean, standard deviation, and percentage of symptomatic responses) for the total sample and subsamples female and male.

\begin{tabular}{|c|c|c|c|c|c|c|c|c|c|}
\hline \multirow{2}{*}{ Item } & \multicolumn{3}{|c|}{ Total Sample } & \multicolumn{3}{|c|}{ Female } & \multicolumn{3}{|c|}{ Male } \\
\hline & $M$ & $S D$ & $\%$ & $M$ & $S D$ & $\%$ & $M$ & $S D$ & $\%$ \\
\hline 1-I feel more nervous and anxious than usual. & 1.54 & 0.73 & 41.8 & 1.58 & 0.76 & 44.7 & 1.48 & 0.69 & 37.9 \\
\hline 2-I feel afraid for no reason. & 1.12 & 0.37 & 10.5 & 1.11 & 0.31 & 10.5 & 1.14 & 0.44 & 10.3 \\
\hline 3-I get upset easily or feel panicky. & 1.12 & 0.37 & 10.5 & 1.11 & 0.31 & 10.5 & 1.14 & 0.44 & 10.3 \\
\hline 4-I feel like I'm falling apart and going to pieces. & 1.27 & 0.59 & 20.9 & 1.24 & 0.49 & 21.0 & 1.31 & 0.71 & 19.8 \\
\hline 5-I feel that everything is all right and nothing bad will happen. & 2.25 & 0.05 & 70.2 & 2.39 & 1.00 & 76.3 & 2.07 & 1.10 & 62.0 \\
\hline 6-My arms and legs shake and tremble. & 1.28 & 0.60 & 22.4 & 1.29 & 0.57 & 23.7 & 1.28 & 0.65 & 20.6 \\
\hline 7-I am bothered by headaches neck and back pain & 1.66 & 0.69 & 53.7 & 1.74 & 0.72 & 57.9 & 1.55 & 0.63 & 48.3 \\
\hline 8-I feel weak and get tired easily. & 1.45 & 0.63 & 38.8 & 1.47 & 0.60 & 42.1 & 1.41 & 0.68 & 34.4 \\
\hline 9-I feel calm and can sit still easily. & 2.03 & 1.03 & 59.7 & 2.05 & 1.04 & 60.5 & 2.00 & 1.03 & 58.6 \\
\hline 10-I can feel my heart beating fast. & 1.34 & 0.59 & 28.4 & 1.45 & 0.65 & 36.8 & 1.21 & 0.49 & 17.2 \\
\hline $11-$ I am bothered by dizzy spells. & 1.16 & 0.48 & 12.0 & 1.21 & 0.53 & 15.8 & 1.10 & 0.41 & 6.8 \\
\hline 12-I have fainting spells or feel like it. & 1.01 & 0.12 & 1.5 & 1.03 & 0.16 & 2.6 & 1.00 & 0.00 & 0.0 \\
\hline 13-I can breathe in and out easily. & 1.36 & 0.71 & 25.4 & 1.24 & 0.49 & 21.0 & 1.52 & 0.91 & 31.0 \\
\hline 14-I get feelings of numbness and tingling in my fingers \& toes. & 1.34 & 0.59 & 29.9 & 1.42 & 0.64 & 36.8 & 1.24 & 0.51 & 20.6 \\
\hline 15-I am bothered by stomach aches or indigestion. & 1.39 & 0.63 & 31.4 & 1.26 & 0.50 & 23.7 & 1.55 & 0.74 & 41.4 \\
\hline 16-I have to empty my bladder often. & 1.84 & 0.75 & 64.2 & 1.87 & 0.74 & 65.8 & 1.79 & 0.77 & 62.0 \\
\hline 17-My hands are usually dry and warm. & 2.60 & 1.11 & 79.2 & 2.82 & 1.11 & 81.6 & 2.31 & 1.07 & 75.9 \\
\hline 18-My face gets hot and blushes. & 1.94 & 0.87 & 62.7 & 1.97 & 0.89 & 63.1 & 1.90 & 0.86 & 62.0 \\
\hline 19-I fall asleep easily and get a good night's rest. & 1.52 & 0.74 & 38.8 & 1.53 & 0.76 & 39.4 & 1.52 & 0.74 & 37.9 \\
\hline 20-I have nightmares. & 1.40 & 0.68 & 31.4 & 1.42 & 0.64 & 34.2 & 1.38 & 0.73 & 27.5 \\
\hline
\end{tabular}

Table 7. Comparison of the total obtained from the application of SAS in the first $\left(1^{\text {st }}\right)$, second $\left(2^{\text {nd }}\right)$, third $\left(3^{\text {rd }}\right)$ and fourth $\left(4^{\text {th }}\right)$ evaluation moments.

\begin{tabular}{|c|c|c|c|c|c|c|}
\hline \multicolumn{2}{|c|}{ Comparison (moments) } & \multirow{2}{*}{$\begin{array}{c}\text { Mean } \\
41.70\end{array}$} & \multirow{2}{*}{$\frac{\boldsymbol{N}}{219}$} & \multirow{3}{*}{$\frac{\boldsymbol{t}}{3.508}$} & \multirow{3}{*}{$\begin{array}{l}\text { d.f. } \\
218\end{array}$} & \multirow{3}{*}{$\begin{array}{c}\text { Sig. (2-tailed) } \\
0.001^{*}\end{array}$} \\
\hline \multirow{2}{*}{$1^{\text {st }}$ Moment $^{*} 2^{\text {nd }}$ Moment } & $S A S 1^{s t}$ & & & & & \\
\hline & $S A S 2^{\text {nd }}$ & 40.07 & 219 & & & \\
\hline \multirow{2}{*}{$1^{\text {st }}$ Moment ${ }^{*} 3^{\text {rd }}$ Moment } & $S A S 1^{s t}$ & 42.17 & 80 & \multirow{2}{*}{1.297} & \multirow{2}{*}{79} & \multirow{2}{*}{0.198} \\
\hline & $S A S 3^{r d}$ & 40.94 & 80 & & & \\
\hline \multirow{2}{*}{$1^{\text {st }}$ Moment $^{*} 4^{\text {th }}$ Moment } & $S A S 1^{s t}$ & 41.86 & 59 & \multirow{2}{*}{4.614} & \multirow{2}{*}{58} & \multirow{2}{*}{$0.000^{*}$} \\
\hline & $S A S 4^{\text {th }}$ & 36.65 & 59 & & & \\
\hline \multirow{2}{*}{$2^{\text {nd }}$ Moment $^{*} 3^{\text {rd }}$ Moment } & $S A S 2^{\text {nd }}$ & 39.48 & 60 & \multirow{2}{*}{-0.807} & \multirow{2}{*}{59} & \multirow{2}{*}{0.423} \\
\hline & $S A S 3^{r d}$ & 40.19 & 60 & & & \\
\hline \multirow{2}{*}{$2^{\text {nd }}$ Moment $^{*} 4^{\text {th }}$ Moment } & $S A S 2^{\text {nd }}$ & 39.81 & 45 & \multirow{2}{*}{2.342} & \multirow{2}{*}{44} & \multirow{2}{*}{$0.024^{*}$} \\
\hline & $S A S 4^{\text {th }}$ & 37.14 & 45 & & & \\
\hline \multirow{2}{*}{$3^{\text {rd }}$ Moment ${ }^{*} 4^{\text {th }}$ Moment } & $S A S 3^{r d}$ & 39.55 & 33 & \multirow{2}{*}{2.960} & \multirow{2}{*}{32} & \multirow{2}{*}{$0.006^{*}$} \\
\hline & $S A S 4^{\text {th }}$ & 36.48 & 33 & & & \\
\hline
\end{tabular}

\subsection{Comparison of the Anxiety Rates Regarding Socio-Demographic Variables over the Four Moments Evaluated}

We compared the SAS questionnaire total mean, as well as the subscales means regarding socio-demographic variables (age, gender, marital status, type of disease, genetic test result) and we found some significant values.

Thus, with respect to gender variable it was found that women had, over the several moments, significantly higher values than men $(p<0.050)$ for total SAS ques- tionnaire and for motor anxiety subscale $\left(1^{\text {st }}\right.$ moment $)$, for cognitive anxiety subscale ( $1^{\text {st }}, 2^{\text {nd }}$ and $3^{\text {rd }}$ moments), for vegetative anxiety subscale ( $1^{\text {st }}$ and $3^{\text {rd }}$ moment), and for CNS anxiety subscale ( $1^{\text {st }}$ and $2^{\text {nd }}$ moment) as we can see consulting Table 12.

Respecting to the age variable, when compared the mean of CNS anxiety ( $1^{\text {st }}$ moment) we verify that subjects between $61-70$ and $41-50$ have higher values; when compared the motor anxiety subscale mean $\left(2^{\text {nd }}\right.$ and $3^{\text {rd }}$ moment), we found that older subjects (age be- 
Table 8. Comparison of the total means for the SAS motor anxiety subscale in the first $\left(1^{\text {st }}\right)$, second $\left(2^{\text {nd }}\right)$, third $\left(3^{\text {rd }}\right)$ and fourth $\left(4^{\text {th }}\right)$ evaluation moments.

\begin{tabular}{|c|c|c|c|c|c|c|}
\hline Comparison (moments) & Motor Anxiety & Mean & $N$ & $t$ & $d f$ & Sig. (2-tailed) \\
\hline \multirow{2}{*}{$1^{\text {st }}$ Moment ${ }^{*} 2^{\text {nd }}$ Moment } & $M A S A S 1^{s t}$ & 7.02 & 279 & \multirow{2}{*}{2.139} & \multirow{2}{*}{278} & \multirow{2}{*}{$0.033^{*}$} \\
\hline & $M A S A S 2^{\text {nd }}$ & 6.76 & 279 & & & \\
\hline \multirow{2}{*}{$1^{\text {st }}$ Moment ${ }^{*} 3^{\text {rd }}$ Moment } & $M A S A S 1^{s t}$ & 7.16 & 128 & \multirow{2}{*}{3.068} & \multirow{2}{*}{127} & \multirow{2}{*}{$0.003^{*}$} \\
\hline & $M A S A S 3^{r d}$ & 6.57 & 128 & & & \\
\hline \multirow{2}{*}{$1^{\text {st }}$ Moment $^{*} 4^{\text {th }}$ Moment } & $M A S A S 1^{s t}$ & 6.92 & 66 & \multirow{2}{*}{1.878} & \multirow{2}{*}{65} & \multirow{2}{*}{0.065} \\
\hline & $M A S A S 4^{\text {th }}$ & 6.45 & 66 & & & \\
\hline \multirow{2}{*}{$2^{\text {nd }}$ Moment ${ }^{*} 3^{\text {rd }}$ Moment } & $M A S A S 2^{\text {nd }}$ & 6.97 & 118 & \multirow{2}{*}{2.096} & \multirow{2}{*}{117} & \multirow{2}{*}{$0.038^{*}$} \\
\hline & $M A S A S 3^{r d}$ & 6.61 & 118 & & & \\
\hline \multirow{2}{*}{$2^{\text {nd }}$ Moment ${ }^{*} 4^{\text {th }}$ Moment } & $M A S A S 2^{\text {nd }}$ & 6.78 & 50 & \multirow{2}{*}{0.678} & \multirow{2}{*}{49} & \multirow{2}{*}{0.501} \\
\hline & $M A S A S 4^{\text {th }}$ & 6.54 & 50 & & & \\
\hline \multirow{2}{*}{$3^{\text {rd }}$ Moment ${ }^{*} 4^{\text {th }}$ Moment } & $M A S A S 3^{r d}$ & 6.38 & 45 & \multirow{2}{*}{-0.788} & \multirow{2}{*}{44} & \multirow{2}{*}{0.435} \\
\hline & $M A S A S 4^{\text {th }}$ & 6.62 & 45 & & & \\
\hline
\end{tabular}

Table 9. Comparison of the total means for the SAS cognitive anxiety subscale in the first $\left(1^{\text {st }}\right)$, second $\left(2^{\text {nd }}\right)$, third $\left(3^{\text {rd }}\right)$ and fourth $\left(4^{\text {th }}\right)$ evaluation moments.

\begin{tabular}{|c|c|c|c|c|c|c|}
\hline Comparison (moments) & Cognitive Anxiety & Mean & $N$ & $t$ & $d f$ & Sig. (2-tailed) \\
\hline \multirow{2}{*}{$1^{\text {st }}$ Moment ${ }^{*} 2^{\text {nd }}$ Moment } & $C A S A S 1^{s t}$ & 8.52 & 283 & \multirow{2}{*}{4.468} & \multirow{2}{*}{282} & \multirow{2}{*}{$0.000^{*}$} \\
\hline & $C A S A S 2^{\text {nd }}$ & 7.85 & 283 & & & \\
\hline \multirow{2}{*}{$1^{\text {st }}$ Moment ${ }^{*} 3^{\text {rd }}$ Moment } & $C A S A S 1^{s t}$ & 8.33 & 131 & \multirow{2}{*}{0.890} & \multirow{2}{*}{130} & \multirow{2}{*}{0.375} \\
\hline & $C A S A S 3^{r d}$ & 8.10 & 131 & & & \\
\hline \multirow{2}{*}{$1^{\text {st }}$ Moment ${ }^{*} 4^{\text {th }}$ Moment } & $C A S A S 1^{s t}$ & 8.57 & 67 & \multirow{2}{*}{3.470} & \multirow{2}{*}{66} & \multirow{2}{*}{$0.001^{*}$} \\
\hline & $C A S A S 4^{\text {th }}$ & 7.30 & 67 & & & \\
\hline \multirow{2}{*}{$2^{\text {nd }}$ Moment ${ }^{*} 3^{\text {rd }}$ Moment } & $C A S A S 2^{\text {nd }}$ & 7.84 & 116 & \multirow{2}{*}{-0.830} & \multirow{2}{*}{115} & \multirow{2}{*}{0.408} \\
\hline & $C A S A S 3^{r d}$ & 8.05 & 116 & & & \\
\hline \multirow{2}{*}{$2^{\text {nd }}$ Moment ${ }^{*} 4^{\text {th }}$ Moment } & $C A S A S 2^{\text {nd }}$ & 7.96 & 50 & \multirow{2}{*}{1.537} & \multirow{2}{*}{49} & \multirow{2}{*}{0.131} \\
\hline & $C A S A S 4^{\text {th }}$ & 7.42 & 50 & & & \\
\hline \multirow{2}{*}{$3^{\text {rd }}$ Moment ${ }^{*} 4^{\text {th }}$ Moment } & $C A S A S 3^{r d}$ & 7.93 & 45 & \multirow{2}{*}{1.375} & \multirow{2}{*}{44} & \multirow{2}{*}{0.176} \\
\hline & $C A S A S 4^{\text {th }}$ & 7.38 & 45 & & & \\
\hline
\end{tabular}

Table 10. Comparison of the total means for the SAS vegetative anxiety subscale in the first $\left(1^{\text {st }}\right)$, second $\left(2^{\text {nd }}\right)$, third $\left(3^{\text {rd }}\right)$ and fourth $\left(4^{\text {th }}\right)$ evaluation moments.

\begin{tabular}{|c|c|c|c|c|c|c|}
\hline Comparison (moments) & Vegetative Anxiety & Mean & $N$ & $t$ & $d f$ & Sig. (2-tailed) \\
\hline \multirow{2}{*}{$1^{\text {st }}$ Moment $^{*} 2^{\text {nd }}$ Moment } & $V A S A S 1^{s t}$ & 14.70 & 274 & \multirow{2}{*}{2.012} & \multirow{2}{*}{273} & \multirow{2}{*}{$0.045^{*}$} \\
\hline & $V A S A S 2^{\text {nd }}$ & 14.39 & 274 & & & \\
\hline \multirow{2}{*}{$1^{\text {st }}$ Moment ${ }^{*} 3^{\text {rd }}$ Moment } & $V A S A S 1^{s t}$ & 14.79 & 129 & \multirow{2}{*}{0.752} & \multirow{2}{*}{128} & \multirow{2}{*}{0.454} \\
\hline & $V A S A S 3^{r d}$ & 14.60 & 129 & & & \\
\hline \multirow{2}{*}{$1^{\text {st }}$ Moment $^{*} 4^{\text {th }}$ Moment } & $V A S A S 1^{s t}$ & 14.85 & 65 & \multirow{2}{*}{2.672} & \multirow{2}{*}{64} & \multirow{2}{*}{$0.010^{*}$} \\
\hline & $V A S A S 4^{\text {th }}$ & 13.92 & 65 & & & \\
\hline \multirow{2}{*}{$2^{\text {nd }}$ Moment $^{*} 3^{\text {rd }}$ Moment } & $V A S A S 2^{\text {nd }}$ & 14.54 & 113 & \multirow{2}{*}{-0.664} & \multirow{2}{*}{112} & \multirow{2}{*}{0.508} \\
\hline & $V A S A S 3^{r d}$ & 14.71 & 113 & & & \\
\hline \multirow{2}{*}{$2^{\text {nd }}$ Moment $^{*} 4^{\text {th }}$ Moment } & $V A S A S 2^{\text {nd }}$ & 14.24 & 50 & \multirow{2}{*}{0.768} & \multirow{2}{*}{49} & \multirow{2}{*}{0.446} \\
\hline & $V A S A S 4^{t h}$ & 13.96 & 50 & & & \\
\hline \multirow{2}{*}{$3^{\text {rd }}$ Moment ${ }^{*} 4^{\text {th }}$ Moment } & $V A S A S 3^{\text {rd }}$ & 14.32 & 44 & \multirow{2}{*}{2.048} & \multirow{2}{*}{43} & \multirow{2}{*}{$0.047^{*}$} \\
\hline & $V A S A S 4^{t h}$ & 13.66 & 44 & & & \\
\hline
\end{tabular}


Table 11. Comparison of the total means for the SAS CNS anxiety subscale in the first $\left(1^{\text {st }}\right)$, second $\left(2^{\text {nd }}\right)$, third $\left(3^{\text {rd }}\right)$ and fourth $\left(4^{\text {th }}\right)$ evaluation moments.

\begin{tabular}{|c|c|c|c|c|c|c|}
\hline Comparison (moments) & CNS Anxiety & Mean & $N$ & $t$ & $d f$ & Sig. (2-tailed) \\
\hline \multirow{2}{*}{$1^{\text {st }}$ Moment ${ }^{*} 2^{\text {nd }}$ Moment } & CNSSAS $1^{s t}$ & 3.42 & 285 & \multirow{2}{*}{1.696} & \multirow{2}{*}{284} & \multirow{2}{*}{0.091} \\
\hline & CNSSAS $2^{\text {nd }}$ & 3.29 & 285 & & & \\
\hline \multirow{2}{*}{$1^{\text {st }}$ Moment ${ }^{*} 3^{\text {rd }}$ Moment } & $C N S S A S 1^{s t}$ & 3.54 & 132 & \multirow{2}{*}{2.581} & \multirow{2}{*}{131} & \multirow{2}{*}{$0.011^{*}$} \\
\hline & CNSSAS $3^{r d}$ & 3.20 & 132 & & & \\
\hline \multirow{2}{*}{$1^{\text {st }}$ Moment $^{*} 4^{\text {th }}$ Moment } & $C N S S A S 1^{s t}$ & 3.36 & 67 & \multirow{2}{*}{2.580} & \multirow{2}{*}{66} & \multirow{2}{*}{$0.012^{*}$} \\
\hline & CNSSAS $4^{\text {th }}$ & 2.93 & 67 & & & \\
\hline \multirow{2}{*}{$2^{\text {nd }}$ Moment ${ }^{*} 3^{\text {rd }}$ Moment } & $C N S S A S 2^{\text {nd }}$ & 3.37 & 118 & \multirow{2}{*}{1.185} & \multirow{2}{*}{117} & \multirow{2}{*}{0.238} \\
\hline & $C N S S A S 3^{r d}$ & 3.25 & 118 & & & \\
\hline \multirow{2}{*}{$2^{\text {nd }}$ Moment $^{*} 4^{\text {th }}$ Moment } & $C N S S A S 2^{\text {nd }}$ & 3.04 & 50 & \multirow{2}{*}{0.118} & \multirow{2}{*}{49} & \multirow{2}{*}{0.907} \\
\hline & CNSSAS $4^{\text {th }}$ & 3.02 & 50 & & & \\
\hline \multirow{2}{*}{$3^{\text {rd }}$ Moment ${ }^{*} 4^{\text {th }}$ Moment } & CNSSAS $3^{\text {rd }}$ & 2.93 & 45 & \multirow{2}{*}{-0.133} & \multirow{2}{*}{44} & \multirow{2}{*}{0.895} \\
\hline & CNSSAS $4^{\text {th }}$ & 2.96 & 45 & & & \\
\hline
\end{tabular}

Table 12. Comparison between SAS total and subscales mean values regarding variable gender over the several moments.

\begin{tabular}{|c|c|c|c|c|c|}
\hline \multicolumn{2}{|l|}{ Total Scores SAS } & \multirow{3}{*}{$\begin{array}{l}\text { Mean } \\
43.47 \\
39.62\end{array}$} & \multirow{3}{*}{$\begin{array}{c}\boldsymbol{N} \\
378 \\
275\end{array}$} & \multirow{3}{*}{$\begin{array}{c}\boldsymbol{F} \\
32.247\end{array}$} & \multirow{3}{*}{$\begin{array}{l}\text { Sig. } \\
0.000\end{array}$} \\
\hline $1^{\text {st }}$ moment (SAS $\left.1^{\text {st }}\right)$ & Female & & & & \\
\hline & Male & & & & \\
\hline \multirow[t]{2}{*}{$2^{\text {nd }}$ moment (SAS $\left.2^{\text {nd }}\right)$} & Female & 41.36 & 135 & \multirow{2}{*}{6.680} & \multirow{2}{*}{0.010} \\
\hline & Male & 38.57 & 97 & & \\
\hline \multirow[t]{2}{*}{$3^{\text {rd }}$ moment (SAS $\left.3^{\text {rd }}\right)$} & Female & 42.97 & 48 & \multirow{2}{*}{4.651} & \multirow{2}{*}{0.034} \\
\hline & Male & 38.41 & 37 & & \\
\hline \multicolumn{2}{|l|}{ SAS Subscales Scores } & Mean & $N$ & $F$ & Sig \\
\hline \multirow{2}{*}{ Motor Anxiety $1^{\text {st }}$ moment (MASAS $1^{\text {st }}$ ) } & Female & 7.26 & 390 & \multirow{2}{*}{18.927} & \multirow{2}{*}{0.000} \\
\hline & Male & 6.53 & 278 & & \\
\hline \multirow{2}{*}{ Cognitive anxiety $1^{\text {st }}$ moment (CASAS $1^{\text {st }}$ ) } & Female & 8.75 & 390 & \multirow{2}{*}{14.430} & \multirow{2}{*}{0.000} \\
\hline & Male & 8.01 & 278 & & \\
\hline \multirow{2}{*}{ Cognitive anxiety $2^{\text {nd }}$ moment (CASAS $\left.2^{\text {nd }}\right)$} & Female & 8.15 & 169 & \multirow{2}{*}{5.797} & \multirow{2}{*}{0.017} \\
\hline & Male & 7.50 & 121 & & \\
\hline \multirow{2}{*}{ Cognitive anxiety $3^{\text {rd }}$ moment (CASAS $3^{\text {rd }}$ ) } & Female & 8.50 & 76 & \multirow{2}{*}{4.555} & \multirow{2}{*}{0.035} \\
\hline & Male & 7.60 & 57 & & \\
\hline \multirow{2}{*}{ Vegetative anxiety $1^{\text {st }}$ moment (VASAS $1^{\text {st }}$ ) } & Female & 15.26 & 384 & \multirow{2}{*}{26.853} & \multirow{2}{*}{0.000} \\
\hline & Male & 14.09 & 279 & & \\
\hline \multirow{2}{*}{ Vegetative anxiety $3^{\text {rd }}$ moment (VASAS $3^{\text {rd }}$ ) } & Female & 15.16 & 76 & \multirow{2}{*}{5.095} & \multirow{2}{*}{0.026} \\
\hline & Male & 13.98 & 57 & & \\
\hline \multirow{2}{*}{ CNS anxiety $1^{\text {st }}$ moment $\left(\right.$ CNSSAS $\left.1^{\text {st }}\right)$} & Female & 3.53 & 392 & \multirow{2}{*}{12.899} & \multirow{2}{*}{0.000} \\
\hline & Male & 3.15 & 280 & & \\
\hline \multirow{2}{*}{ CNS anxiety $2^{\text {nd }}$ moment (CNSSAS $\left.2^{\text {nd }}\right)$} & Female & 3.48 & 170 & 6622 & 0011 \\
\hline & Male & 3.07 & 121 & 0.022 & 0.011 \\
\hline
\end{tabular}

tween 61 and 80 years) are those with higher average (Table 13).

Concerning marital status, it was found significant differences, at $1^{\text {st }}$ moment, for vegetative anxiety subscale $(\mathrm{t}$ $=2.996 ; \mathrm{df}=4 ; \mathrm{p}=0.018)$ : widow individuals had the highest average and, at $3^{\text {rd }}$ moment, six months after disclosure, married subjects had the highest averages in the SAS total mean (Table 14).
Finally, it was found significant values when comparing the total SAS and subscales SAS means with the variable type of disease.

As shown by the observation of Table 15, at $1^{\text {st }}$ moment, only the CNS anxiety subscale presents significant values, indicating that the subjects who performed the HD PST as having the highest values.

At $2^{\text {nd }}, 3^{\text {rd }}$ and $4^{\text {th }}$ moments, after disclosure, MJD sub- 
Table 13. Comparison between SAS total and subscales mean values regarding variable age over the several moments.

\begin{tabular}{|c|c|c|c|c|c|c|}
\hline Moments & & & Mean & $\mathbf{N}$ & $\mathbf{F}$ & Sig. \\
\hline $1^{\text {st }}$ moment & $\begin{array}{l}\text { CNS anxiety } \\
\text { CNSSAS }^{\text {st }}\end{array}$ & $\begin{array}{l}17-30 \\
31-40 \\
41-50 \\
51-60 \\
61-70 \\
71-80\end{array}$ & $\begin{array}{l}3.38 \\
3.16 \\
3.60 \\
3.51 \\
3.94 \\
3.43\end{array}$ & $\begin{array}{c}262 \\
229 \\
73 \\
55 \\
33 \\
7\end{array}$ & 2.880 & 0.014 \\
\hline $2^{\text {nd }}$ moment & $\begin{array}{l}\text { Motor Anxiety } \\
\text { MASAS } 2^{\text {nd }}\end{array}$ & $\begin{array}{l}17-30 \\
31-40 \\
41-50 \\
51-60 \\
61-70 \\
71-80\end{array}$ & $\begin{array}{l}6.82 \\
6.38 \\
7.18 \\
6.77 \\
8.06 \\
7.77\end{array}$ & $\begin{array}{c}111 \\
99 \\
28 \\
26 \\
16 \\
3\end{array}$ & 2.247 & 0.050 \\
\hline $3^{\text {rd }}$ moment & $\begin{array}{l}\text { Motor Anxiety } \\
\text { MASAS } 3^{\text {rd }}\end{array}$ & $\begin{array}{l}17-30 \\
31-40 \\
41-50 \\
51-60 \\
61-70 \\
71-80\end{array}$ & $\begin{array}{l}6.35 \\
6.00 \\
6.87 \\
7.50 \\
8.29 \\
8.00\end{array}$ & $\begin{array}{c}48 \\
45 \\
15 \\
14 \\
7 \\
1\end{array}$ & 2.433 & 0.039 \\
\hline
\end{tabular}

Table 14. Comparison between the mean values of the SAS subscales regarding the variable marital status over the several moments.

\begin{tabular}{|c|c|c|c|c|c|c|}
\hline Moments & Subscal & & Mean & $\mathbf{N}$ & $\mathbf{F}$ & Sig. \\
\hline \multirow{4}{*}{$1^{\text {st }}$ moment } & \multirow{4}{*}{ Vegetative anxiety } & Single & 14.52 & 336 & \multirow{4}{*}{2.996} & \multirow{4}{*}{0.018} \\
\hline & & Married & 14.94 & 294 & & \\
\hline & & Divorced & 13.92 & 12 & & \\
\hline & & Widow & 17.10 & 10 & & \\
\hline \multirow{4}{*}{$3^{\text {rd }}$ moment } & \multirow{4}{*}{ Total SAS } & Single & 38.48 & 46 & \multirow{4}{*}{4.230} & \multirow{4}{*}{0.018} \\
\hline & & Married & 44.24 & 38 & & \\
\hline & & Divorced & 0 & 0 & & \\
\hline & & Widow & 0 & 0 & & \\
\hline
\end{tabular}

Table 15. Comparison between the mean values of the SAS totals and subscales with the variable type of disease over the several moments.

\begin{tabular}{|c|c|c|c|c|c|c|}
\hline Moments & \multicolumn{2}{|c|}{ Subscales/Totals } & Mean & $\mathbf{N}$ & $\mathbf{F}$ & Sig. \\
\hline $1^{\text {st }}$ moment & CNS anxiety & $\begin{array}{c}F A P \\
M J D \\
H D\end{array}$ & $\begin{array}{l}3.34 \\
2.75 \\
3.66\end{array}$ & $\begin{array}{c}574 \\
8 \\
90\end{array}$ & 3.019 & 0.050 \\
\hline \multirow{2}{*}{$2^{\text {nd }}$ moment } & Total SAS & $\begin{array}{c}F A P \\
M J D \\
H D\end{array}$ & $\begin{array}{l}39.80 \\
51.25 \\
41.17\end{array}$ & $\begin{array}{c}195 \\
4 \\
33\end{array}$ & \multirow{2}{*}{$\begin{array}{l}4.194 \\
3.839\end{array}$} & \multirow{2}{*}{$\begin{array}{l}0.016 \\
0.023\end{array}$} \\
\hline & Cognitive anxiety & $\begin{array}{c}F A P \\
M J D \\
H D\end{array}$ & $\begin{array}{c}7.83 \\
11.00 \\
7.82\end{array}$ & $\begin{array}{c}247 \\
4 \\
39\end{array}$ & & \\
\hline $3^{\text {rd }}$ moment & Motor Anxiety & $\begin{array}{c}F A P \\
M J D \\
H D\end{array}$ & $\begin{array}{l}6.28 \\
8.50 \\
7.48\end{array}$ & $\begin{array}{c}104 \\
4 \\
25\end{array}$ & 5.154 & 0.007 \\
\hline $4^{\text {th }}$ moment & CNS anxiety & $\begin{array}{c}F A P \\
M J D \\
H D\end{array}$ & $\begin{array}{l}2.73 \\
5.00 \\
3.25\end{array}$ & $\begin{array}{c}49 \\
2 \\
16\end{array}$ & 5.183 & 0.008 \\
\hline
\end{tabular}

jects are those having significantly higher values in SAS subscales and also in the SAS total score, three weeks after disclosure, presenting total scores ( $>40$ points) revealing clinical anxiety.

\subsection{Predictors of the Self-Rating Anxiety Scale of Zung (SAS)}

We intend to know the predictive value of some socio- demographic and other variables that could take an explicative character to the values found in the SAS scale over the four evaluation moment sand for the three studied diseases.

Thus, we performed the multiple linear regression analysis using stepwise estimation method [22] for the total scores of the SAS scale, as well as for cognitive, motor, vegetative and CNS SAS subscales. We consid- 
ered the socio-demographic variables as independent variables.

Analyzing Table 16, we can see that gender is the variable that has a higher predictive value in the regression equation explaining $4 \%$ of the dependent variable Total SAS variance, at 1 st moment. The final equation is made by the independent variables gender and test result $\left(\mathrm{R}^{2}=0.51, \mathrm{~F}=17.849, \mathrm{df}=2, \mathrm{p}=0.000\right)$ explaining, overall, $5.1 \%$ of the total SAS score variance, at $1^{\text {st }}$ moment (Table 16).

It was also found that the independent variable gender is the one with the most predictive power in the regression equation, explaining $2 \%$ of the dependent variable Total SAS variance, at $2^{\text {nd }}$ moment; the final equation is made by the independent variables gender, type of disease and test result $\left(\mathrm{R}^{2}=0.06, \mathrm{~F}=4.644\right.$, $\mathrm{df}=3, \mathrm{p}=$ 0.004 ) explaining, overall, $6 \%$ of the total SAS score variation, at $2^{\text {nd }}$ moment (Table 17).

Analyzing Table 18, we can see that the independent variable, marital status, shows the highest predictive value in the regression equation, explaining $9 \%$ of the dependent variable Total SAS variance, at $3^{\text {rd }}$ moment; the final equation is made by the independent variables marital status, gender and test result $\left(\mathrm{R}^{2}=0.21, \mathrm{~F}=\right.$ $6.804, \mathrm{df}=3, \mathrm{p}=0.000$ ) which explain, overall, $21 \%$ of the total SAS score variation, at $3^{\text {rd }}$ moment (Table 18).

Then, we conducted linear regression analyzes for all SAS subscales and for all the evaluation moments considered. These analyze yielded the following significant results:

For the cognitive anxiety subscale, it was found that the independent variable gender was the only one that had predictive value in the regression equation $\left(\mathrm{R}^{2}=\right.$

Table 16. Multiple linear regression analysis for variables predicting the Total SAS $1^{\text {st }}$ moment.

\begin{tabular}{ccccc}
\hline MODEL & VARIABLE & B & SE & $\boldsymbol{\beta}$ \\
\hline $\mathbf{1}$ & Gender & -3.817 & 0.688 & $-0.216^{* *}$ \\
$\mathbf{2}$ & Gender & -3.900 & 0.687 & $-0.220^{* *}$ \\
& Test Result & -1.327 & 0.612 & $-0.084^{*}$ \\
\hline
\end{tabular}

$\mathrm{R}^{2}=0.04$ step $1 ; \Delta \mathrm{R}^{2}=0.05$ step $2 ;{ }^{* *} \mathrm{p}<0.010,{ }^{*} \mathrm{p}<0.050$.

Table 17. Multiple linear regression analysis for variables predicting the Total SAS $2^{\text {nd }}$ moment.

\begin{tabular}{ccccc}
\hline MODEL & VARIABLE & B & SE & $\boldsymbol{\beta}$ \\
\hline \multirow{2}{*}{$\mathbf{1}$} & Gender & -2.520 & 1.114 & $-0.151^{*}$ \\
& Gender & -2.412 & 1.105 & $-0.144^{*}$ \\
$\mathbf{2}$ & Type of Disease & 2.767 & 1.276 & $0.143^{*}$ \\
& Gender & -2.611 & 1.103 & $-0.156^{*}$ \\
\multirow{2}{*}{3} & Type of Disease & 3.161 & 1.283 & 0.164 \\
& Test Result & -2.012 & 1.020 & -0.132 \\
\hline
\end{tabular}

$\mathrm{R}^{2}=0.02$ step $1 ; \Delta \mathrm{R}^{2}=0.04 \operatorname{step} 2 \Delta \mathrm{R}^{3}=0.06$ step $3 ;{ }^{* *} \mathrm{p}<0.010,{ }^{*} \mathrm{p}<$ 0.050 .
Table 18. Multiple linear regression analysis for variables predicting the Total SAS $3^{\text {rd }}$ moment.

\begin{tabular}{ccccc}
\hline MODEL & VARIABLE & B & SE & $\boldsymbol{\beta}$ \\
\hline \multirow{2}{*}{$\mathbf{2}$} & Marital Status & 5.577 & 2.021 & $0.293^{* *}$ \\
& Marital Status & 5.540 & 1.976 & $0.291^{* *}$ \\
$\mathbf{2}$ & Gender & -4.514 & 2,064 & $-0.227^{*}$ \\
& Marital Status & 6.237 & 1.927 & $0.324^{* *}$ \\
\multirow{3}{*}{3} & Gender & -5.565 & 2.034 & $-0.280^{* *}$ \\
& Test Result & -5.589 & 2.153 & $-0.268^{*}$ \\
\hline
\end{tabular}

$\mathrm{R}^{2}=0.09$ step $1, \Delta \mathrm{R}^{2}=0.14$ step $2, \Delta \mathrm{R}^{3}=0.21$ step $3 ;{ }^{* *} \mathrm{p}<0.010,{ }^{*} \mathrm{p}<$ 0.050 .

$0.20, \mathrm{~F}=13.032, \mathrm{df}=1, \mathrm{p}=0.000)$, explaining $2 \%$ of the variance at $1^{\text {st }}$ moment. The same can be said, for the same dependent variable, at $2^{\text {nd }}$ moment, the independent variable gender continued to explained $2 \%$ of the variance $\left(\mathrm{R}^{2}=0.20, \mathrm{~F}=5.756, \mathrm{df}=1, \mathrm{p}=0.017\right)$. Concerning yet this dependent variable, at $3^{\text {rd }}$ moment, we observed that the independent variable type of disease was the only one that explained $4 \%$ of the variance $\left(R^{2}=0,43\right.$, $\mathrm{F}=5,722, \mathrm{df}=1, \mathrm{p}=0,018)$.

It was verified that the independent variable gender was the only one that had predictive value in the regression equation $\left(\mathrm{R}^{2}=0.27, \mathrm{~F}=18.018, \mathrm{df}=1, \mathrm{p}=0.000\right)$, by explaining $3 \%$ of the variance of the dependent variable motor anxiety subscale, at $1^{\text {st }}$ moment. At $3^{\text {rd }}$ moment, we found that the two independent variables type of disease and test results, together, explained $11 \%$ of the dependent variable motor anxiety subscale variance $\left(\mathrm{R}^{2}\right.$ $=0.11, \mathrm{~F}=7.892, \mathrm{df}=2, \mathrm{p}=0.001)$.

For the dependent variable vegetative anxiety subscale, at $1^{\text {st }}$ moment, the independent variables gender, test result, and marital status explained $6 \%$ of its variance $\left(\mathrm{R}^{2}\right.$ $=0.06, \mathrm{~F}=12.670, \mathrm{df}=3, \mathrm{p}=0.000)$; at $3^{\text {rd }}$ moment, the independent variables age and gender, together, explained $8 \%$ of the variance of this same dependent variable $\left(\mathrm{R}^{2}=0.08, \mathrm{~F}=5.610, \mathrm{df}=2, \mathrm{p}=0.005\right)$; finally, at $4^{\text {th }}$ moment, the independent variable marital status was the one that explained $9 \%$ of the variance.

At last, regarding the dependent variable CNS anxiety subscale, at $1^{\text {st }}$ moment, the dependent variables gender and age, together, explained $3 \%\left(\mathrm{R}^{2}=0.03, \mathrm{~F}=8.817\right.$, df $=2, \mathrm{p}=0.000)$ of its variance; at $2^{\text {nd }}$ moment, the independent variable gender, explained $2 \%$ of its variance $\left(\mathrm{R}^{2}=0.02, \mathrm{~F}=4.729, \mathrm{df}=1, \mathrm{p}=0.031\right)$; finally, at $4^{\text {th }}$ moment, the independent variable type of disease, explained $11 \%$ of its variance $\left(\mathrm{R}^{2}=0.11, \mathrm{~F}=8.191\right.$, $\mathrm{df}=1$, $\mathrm{p}=0.006)$.

\section{DISCUSSION}

We have found that the number of patients leaving the protocol over one year was quite high and this can be the principal limitation of this study; thus, this can bias the conclusions we draw from the data obtained. We found 
that, proportionally, the number of carriers increases and non carriers decreases over protocol, i.e., the carriers remain in the protocol more than non carriers; therefore, it is necessary to take into account this point as one of the limitations of this study.

The descriptive analysis, such as a previous study of measurement of scale to Portuguese population [19], revealed that female had higher values of anxiety symptoms. The pre-test $\left(1^{\text {st }}\right.$ moment) recorded higher values for both genders, although for women values were indicative of clinical anxiety (score $\geq 40$ ) and men were on the border between normal and pathological anxiety. For both groups it can be stated that anxiety decreased over four assessment moments.

We also obtained results quite acceptable for internal consistency, since the avalues were always, for all moments and groups considered, very close to 0.80 , leading us to conclude that this instrument is reliable for the studied population.

By examining the 20 items scale, we find that women, in the pre-test $\left(1^{\text {st }}\right.$ moment), revealed a higher level of restlessness, pessimism and fear, and a greater pain associated with the presence of a higher generalized tension (head, neck and back); these findings seem to corroborate the presence of the total scores inducing anxiety symptoms, even before the completion of the TPS, as said in previous paragraph. Higher values of anxiety symptoms at the beginning of PST, in women, could mean that pre-test $\left(1^{\text {st }}\right.$ moment $)$ itself may bea trigger of anxiety disturbance, as well as other studies have mentioned, supporting the need for psychological support since the beginning of the genetic counseling PST process $[23,24]$.

Three weeks after PST disclosure ( $2^{\text {nd }}$ moment), women continued to show a greater presence of items answered with options-inducing presence of anxiety symptoms, in particular, revealing more likely to present sleep disorders.

These data, i.e., the reduction of anxiety score during the protocol (mostly, from $1^{\text {st }}$ moment to $2^{\text {nd }}$ moment, first post-test immediately after the PST communication), also seem to indicate that the PST brings advantages in reducing the uncertainty and self control effects for the disease to which the at-risk individual decides to make the test $[15,16,25]$.

Considering the anxiety total scores and subscales values regarding socio-demographic variables, some significant results were found:

Thus, with respect to gender variable it was found that women had, over the several moments, significantly higher values than men for total SAS questionnaire and for motor anxiety subscale ( $1^{\text {st }}$ moment), for cognitive anxiety subscale $\left(1^{\text {st }}, 2^{\text {nd }}\right.$ and $3^{\text {rd }}$ moments $)$, for vegetative anxiety subscale ( $1^{\text {st }}$ and $3^{\text {rd }}$ moment), and for CNS anxi- ety subscale ( $1^{\text {st }}$ and $2^{\text {nd }}$ moment $)$.

Respecting to the age variable, when compared the mean of CNS anxiety ( $1^{\text {st }}$ moment) we verify that subjects between 61 - 70 and 41 - 50 have higher values; when compared the motor anxiety subscale mean $\left(2^{\text {nd }}\right.$ and $3^{\text {rd }}$ moment), we found that older subjects(age between 61 and 80 years) are those with higher average. This can be explained, first, according to the SAS scale normalization studies for the portuguese population, there is a greater tendency for older individuals present higher values of anxiety [26]; second, the age of these subjects (between 40 and 51 years) is approaching the age mean considered to the beginning of this late on-set diseases first symptoms, that can lead to higher anxiety values.

Considering marital status, we found a tendency for widow subjects had the highest average in the vegetative anxiety subscale at pre-test $\left(1^{\text {st }}\right.$ moment $)$; this result seems to point to the hypothesis that people at risk and more alone may have greater tendency for a higher level of anxiety symptoms. Widowhood maybe relates to the perceived lack of effective care, by becoming more difficult the existence of a future caregiver. The fact that, in the divorced group, we did not observe the same trend, canbe explained with the age factor, i.e., widows tend to be older people. After 6 months of PST disclosure $\left(3^{\text {rd }}\right.$ moment), the married subjects group had higher total anxiety, compared with the single individuals (note that this moment assessed only subjects with these two marital status); therefore, it may be the existence of a partner or objectives of having a child, significant factors to induce higher values of anxiety, since it was widely studied the importance of partners in the at-risk and/or ill patients for HD [8,27].

Finally, when we compared the total scores and subscales means regarding the type of disease variable, we found significant values for the CNS anxiety subscale, in the pre-test, for HD; this may indicate greater anxiety for those at risk for this disease, given the severity of their clinical condition. This may also be related to some HD carriers psychopathological symptoms, that may already be manifesting at the beginning of the PST protocol $\left(1^{\text {st }}\right.$ moment). This aspect concerning the disease severity might explain why, at all post-test moments $\left(2^{\text {nd }}, 3^{\text {rd }}\right.$ and $4^{\text {th }}$ moments), the HD subjects continued to reveal superior anxiety results, only being surpassed by the MJD subjects (however, MJD group were not significant). Subjects who performed the test for FAP showed lower values, perhaps because they have hope on the drug treatment in the near future or in the currently available solution, the liver transplantation, in order to wage disease progression, both solutions nonexistent for HD or MJD [3,28].

Regarding test result variable, there were no statisti- 
cally significant results, as previous studies were indicated for HD: knowledge of carrier or non-carrier status does not seem to bring a negative psychological impact on individuals [7,8,28].

Several studies have indicated the importance of the socio-demographic variables predictive character for population that performs the PST [3,6-8,16,28] for the establishment of more effective interventions in those individuals identified as vulnerable. This study identified variables such as gender, type of disease, marital status as having some predictive value with respect to what can be expected about future anxiety symptoms presented along the several assessed moments. For this reason, the need for a personalized and careful monitoring to each individual who performs a PST protocol remains a substantial ethical principle in conducting such genetic tests [2, 3].

\section{CONCLUSIONS}

We found a decrease in mean values over the four evaluations moments regarding total scores obtained by applying the Self-Ranting Anxiety Scale of Zung (SAS), evidencing that subjects have higher values before presymptomatic test $\left(1^{\text {st }}\right.$ moment $)$ than in the several posttesting moments $\left(2^{\text {nd }}, 3^{\text {rd }}\right.$ and $4^{\text {th }}$ moments $)$, mainly a year after knowing their genetic status. However, for the female population, the SAS means scores revealed a result of clinical anxiety ( $>40$ points) from the pre-test $\left(1^{\text {st }}\right.$ moment), only decreasing to non clinical scores a year after PST disclosure ( $4^{\text {th }}$ moment).

The inherent characteristics of each disease here studied, as well as the knowledge of the genetic status - to be or not to be a carrier - do not appear to significantly influence the presence of anxiety disorder. However, we find a lower trend in subject's average who took the PST for FAP.

The variables gender, age and marital status, showed an oscillating weight in the anxiety scores verifying that female has higher values, as well as the older subjects or those who are closer to the beginning of the first symptoms; widows also had the highest anxiety scores.

Although, from a clinical point of view, we have not found values indicating anxiety disorder, we can conclude however that pre-symptomatic test for studying diseases causes a considerable anxiety level, since the averages were always very close to the cutoff point of the SAS.

\section{REFERENCES}

[1] International Huntington Association and World Federation of Neurology Research Group on Huntington's Disease (1994) Guidelines for the molecular genetics predictive test in Huntington's disease. Journal of Medical Ge- netics, 31, 555-559. doi:10.1136/jmg.31.7.555

[2] Sequeiros, J. (1996) Aconselhamento genético e teste preditivo na Doença de Machado-Joseph. In: Sequeiros, J., Ed., O Teste Preditivo da Doença de Machado-Joseph, UnIGENe, IBMC, Porto.

[3] Paneque, H.M., Prieto, A.L., Reynaldo, R.R., Cruz, M.T., Santos, F.N., Almaguer, M.L., et al. (2007) Psychological aspects of presymptomatic diagnosis of spinocerebellar ataxia type 2 in Cuba. Journal of Community Genetics, 10, 132-139. doi:10.1159/000101754

[4] Lerman, C. (1997) Psychological aspects of genetic testing: Introduction to the special issue. Health Psychology, 16, 3-7. doi: $10.1037 / \mathrm{h} 0092702$

[5] Decruyenaere, M., Evers-Kiebooms, G. and Van Den Berghe, H. (1997) Non-participation in predictive testing for Huntington's Disease: Individual decision-making, personality and avoidant behavior in the family. European Journal of Human Genetics, 5, 351-363.

[6] Almqvist, E.W., Bloch, M. and Hayden, M. (1999) A worldwide assessment of the frequency of suicide, suicide attempts, or psychiatric hospitalization after predictive testing for Huntington Disease. American Journal of Human Genetics, 64, 1293-1304. doi:10.1086/302374

[7] Codori, A., Slavney, P.R. and Brandt, J. (1997) Predictors of psychological adjustment to genetic testing of Huntington's Disease. Health Psychology, 16, 36-50. doi:10.1037/0278-6133.16.1.36

[8] Tibben, A., Timman, R., Bannink, E. and Duivenvoorden, H. (1997) Three years follow-up after presymptomatic testing for Huntington's Disease in tested individuals and partners. Health Psychology, 16, 20-35. doi:10.1037/0278-6133.16.1.20

[9] Sequeiros, J. (1996) Genética clássica e genética molecular na Doença de Machado-Joseph. In: Sequeiros, J., Ed., O Teste Preditivo da Doença de Machado-Joseph, UnIGENe, IBMC, Porto, 1996.

[10] Saraiva, M.J. and Costa, P. (1986) Familial amyloidotic polyneuropathy, Portuguese type: Phenotype and genotype. In: Sales Luís, M.L., Ed., Symposiumon Peripheral Neuropathies, Lisboa, 207-212.

[11] Lopes, A. and Fleming, M. (1996) Doença somática e organização psíquica: Reflexões a partir da Polineuropatia Amiloidótica Familiar. Revista Portuguesa de Psicanálise, 15, 93-100.

[12] Lopes, A. and Fleming, M. (1998) Aspectos psicológicos da Polineuropatia Amiloidótica Familiar: A trama subterrânea intergeracional. Brotéria Genética, XIX, 183192.

[13] Lêdo, S.O. (2002) Primeiro dia do resto de suas vidas. Alguns aspectos psicológicos da Paramiloidose. MSc Thesis, ISPA, Lisbon.

[14] Leite, A. (2006) Determinantes psicossociais da adesão ao teste pré-sintomático em doenças neurológicas hereditárias de aparecimento tardio. PhD Thesis, Instituto de Ciências Biomédicas Abel Salazar da Universidade do Porto, Porto, 2006.

[15] Paneque, H.M., Lemos, C., Sousa, A., Velázquez, P.L., Fleming, M. and Sequeiros, J. (2009) Role of the disease 
in the psychological impact of pre-symptomatic testing for SCA2 and FAP ATTRV30M: Experience with the disease, kinship and gender of the transmitting parent. Journal of Genetic Counseling, 18, 483-493. doi:10.1007/s10897-009-9240-1

[16] Rolim, L., Leite, A., Ledo, S., Paneque, M., Sequeiros, J. and Fleming, M. (2006) Psychological aspects of presymptomatic testing for Machado-Joseph disease and familial amyloid polyneuropathy type I. Clinical Genetics, 69, 297-305. doi:10.1111/j.1399-0004.2006.00606.x

[17] Sequeiros, J. (1996) História da Doença de MachadoJoseph. In: Sequeiros, J., Ed., O Teste Preditivo da Doença de Machado-Joseph, UnIGENe, IBMC, Porto, 1996.

[18] Coutinho, P. (1996) Aspectos clínicos, história natural e epidemiologia na Doença de Machado-Joseph. In: Sequeiros, J., Ed., O Teste Preditivo da Doença de Machado-Joseph, UnIGENe, IBMC, Porto.

[19] Vaz Serra, A., Ponciano, E. and Relvas, J. (1982) Aferição da escala de auto-avaliação de ansiedade de SAS, numa amostra da população portuguesa-II-Sua avaliação como instrumento de medida. Psiquiatria Clínica, 3, 203213.

[20] Zung, W.A. (1975) Rating instrument for anxiety disorders. Psychosomatics, 12, 371-379.

[21] Norusis, M.J. (2011) PASW statistics 19 guide to data analysis by Inc. SPSS. Pearson, 2011.

[22] Pestana, M.H. and Gageiro, J.N. (1998) Análise de dados para Ciências Sociais-A complementaridade do SPSS. Edições Sílabo, Lisboa.
[23] Sequeiros, J. (1998) Prenatal diagnosis of late-onset diseases. Progresos en Diagnóstico Prenatal, 10, 218-220.

[24] Weil, J. (2003) Psychosocial genetic counseling in the post-nondirective era: A point of view. Journal of $\mathrm{Ge}$ netic Counseling, 12, 199-211. doi:10.1023/A:1023234802124

[25] Almqvist, E.W., Brinkman, R.R., Wiggins, S. and Hayden, M.R. (2003) Canadian collaborative study of predictive testing. Psychological consequences and predictors of adverse events in the first 5 years after predictive testing for Huntington's disease. Clinical Genetics, 64, 300309. doi:10.1034/j.1399-0004.2003.00157.x

[26] Ponciano, E., Vaz Serra, A. and Relvas, J. (1982) Aferição da Escala de Auto-avaliação de Ansiedade de SAS, numa amostra da população portuguesa-I. Resultados da aplicação numa amostra de população normal. Psiquiatria Clínica, 3, 191-202.

[27] Richards, F. and Williams, K. (2004) Impact on couple relationships of predictive testing for Huntington disease: A longitudinal study. American Journal of Medical Genetics, 126, 161-169.

[28] DudokdeWit, A.C., Tibben, A., Duivenvoorden, H.J., Niermeijer, M.F. and Passchier, J. (1998) Predicting adaptation to presymptomatic DNA testing for late onset disorders: Who will experience distress? Rotterdam Leiden Genetics Workgroup. Journal of Medical Genetics, 35, 745754. doi:10.1136/jmg.35.9.745 